\title{
Non-Invasive Adenomatous Component in Carcinoma of Papilla of Vater: An Immunohistochemical Study of Carbohydrate Antigen, CA19-9
}

\author{
Hidemi Yamauchi, Atsushi Nitta, Ryo Ichinohasama $\dagger$ \\ and Tsuneo Namiki* \\ Department of Surgery, *Department of Pathology, Sendai \\ National Hospital, Sendai 983, and †Department of \\ Pathology, Tohoku University Hospital, Sendai 980
}

\begin{abstract}
Yamauchi, H., Nitta, A., Ichinohasama, R. and Namiki, T. Non-Invasive Adenomatous Component in Carcinoma of Papilla of Vater: An Immunohistochemical Study of Carbohydrate Antigen, CA19-9. Tohoku J. Exp. Med., 1989, 159 (1), 81-82 Eleven patients with carcinoma of papilla of Vater accompanied by non-invasive adenomatous component (nac) had good prognosis after surgical resection. These carcinomas were associated with low serum CA199 levels seldom invaded or metastasized to the pancreas, duodenum, lymph node and vein. On the contrary, 12 patients whose carcinomas lacked in 'nac' had poor prognosis after resection. The 'nac' negative carcinomas invaded or metastasised more frequently to the pancreas, duodenum, lymph node and vein. A half of the 'nac' negative carcinoma showed high serum level of CA19-9. A significant different immunohistochemical pattern of CA19-9 was demonstrated between the 'nac' positive and negative carcinomas. The former showed partial, while the latter showed diffuse distribution pattern. —_ carcinoma of papilla of Vater ; non-invasive adenomatous component; immunohistochemistry of Ca19-9; histopathology
\end{abstract}

Non-invasive adenomatous component (nac) associated with the invasive carcinoma of the papilla of Vater was defined in this investigation of the adenoma like glands without stromal invasion such as irregular protrusion of the gland into the surrouding stroma with desmoplastic reaction, cribriform pattern, or papillary proliferation of the gland. In order to scrutinize the difference between the 'nac' negative and positive carcinoma, an immunohistocemical study of CA19-9 was made in 23 carcinomas obtained after pancreatoduodenectomy in the Department of Surgery, Sendai National Hospital, with ABC method (Hsu et al. 1981) employing monoclonal antibody given by Toray Fuji Bionics, Inc., Tokyo. Furthermore, the frequency of cancerous spread to the pancreas, duodenum, lymph node, and vein was also examined histologically.

A photomicrograph of the typical 'nac' is shown in Fig. 1. The 'nac' positive carcinoma showed partial distribution of the CA19-9, while the 'nac' negative carcinoma showed diffuse distribution (Figs. 2, 3 and Table 1). Serum level of CA19-9 measured by EIA method in the 'nac' positive and negative carcinoma was $31.6 \pm 10.9(\mathrm{U} / \mathrm{ml})$ and 300.4 with a range from 9 to 2010, respectively. One out of two carcinomas demonstrated negative distribution of CA19-9 indicated normal serum level of $24(\mathrm{U} / \mathrm{ml})$. Moreover, the 'nac' negative cancinoma invaded or metastasized more to the pancreas, duodenum, lymph

Received August 29, 1989; revision accepted for publication September 5, 1989. 

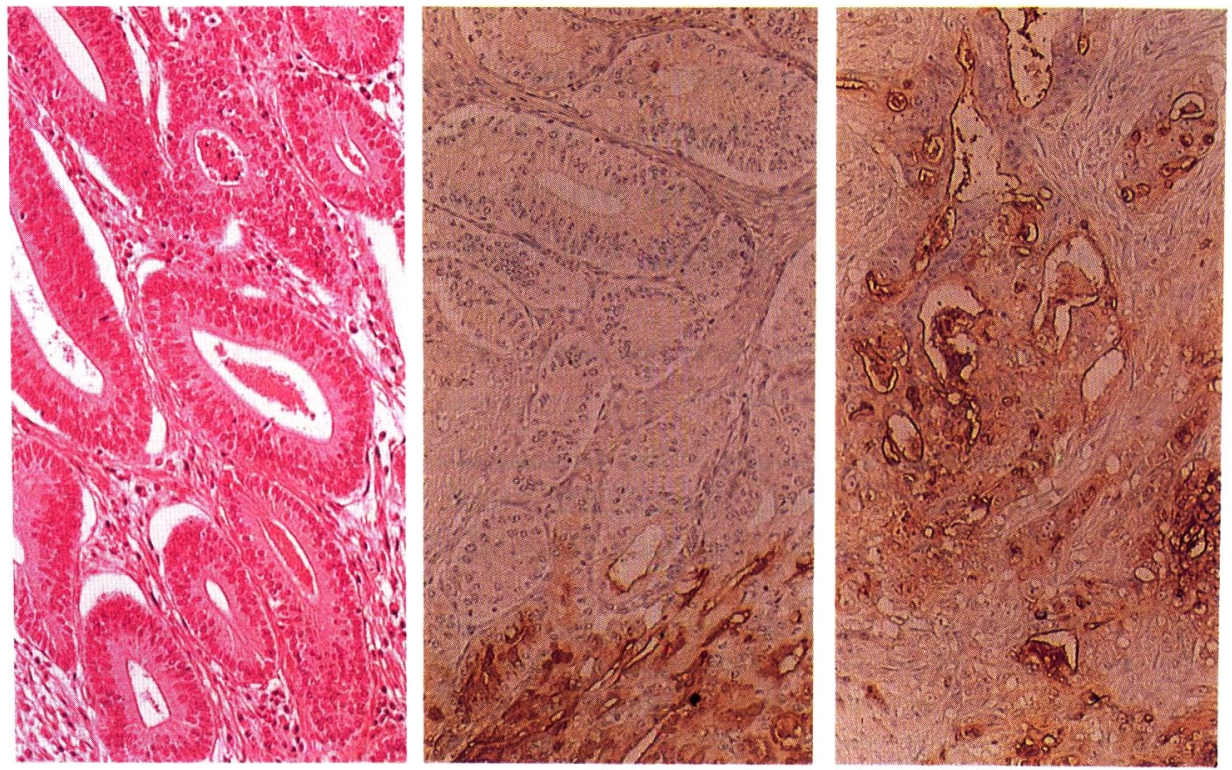

Fig. 1. (left) Non-invasive adenomatous component (HE-stain).

Fig. 2. (middle) Partial distribution of CA19-9 in 'nac' positive carcinoma.

Fig. 3. (right) Diffuse distribution of CA19-9 in 'nac' negative carcinoma.

TABLE 1. State of distribution of CA19-9 and cancer spread

\begin{tabular}{|c|c|c|c|c|c|c|c|}
\hline & \multicolumn{3}{|c|}{ Immunohistochemistry of CA19-9 } & \multirow{3}{*}{$\mathrm{n}$} & \multirow{3}{*}{ panc } & \multirow{3}{*}{$\mathrm{d}$} & \multirow{3}{*}{$\mathrm{v}$} \\
\hline & \multirow{2}{*}{ Negative } & \multicolumn{2}{|c|}{ Positive } & & & & \\
\hline & & Partial & Diffuse & & & & \\
\hline Carcinoma with 'nac' $(n=11)$ & 0 & $11^{\mathrm{A}}$ & $0^{\mathrm{C}}$ & $2^{\mathrm{E}}$ & $1^{\mathrm{G}}$ & $4^{\mathrm{I}}$ & $0^{\mathrm{K}}$ \\
\hline Carcinoma without 'nac' $(n=12)$ & 2 & $2^{\mathrm{B}}$ & $8^{\mathrm{D}}$ & $8^{\mathrm{F}}$ & $6^{\mathrm{H}}$ & $10^{\mathrm{J}}$ & $7^{\mathrm{L}}$ \\
\hline
\end{tabular}

nac, non-invasive adenomatous component; n, nodal involvement; panc, pancreatic invasion; $\mathrm{d}$, duodenal invasion; $\mathrm{V}$, vein invasion.

A vs. B, C vs. D, K vs. L : $p<0.01$; E vs. F, G vs. H, I vs. J : $p<0.1$ (Chi square test with Yates' correction)

node and vein (Table 1). The five year survival rate of the 'nac' positive and negative carcinoma was 75 and $11 \%$, respectively. The difference was statistically significant $(p<0.05)$.

The non-invasive adenomatous component is considered as precancerous condition. However, significantly different clinicopathologic features of these two groups may be based on the different potentialities in cancer development such as adenoma-cancer versus de novo carcinoma (Muto and Adachi 1988).

\section{References}

1) Hsu, S.M., Raine, L. \& Fanger, H. (1981) Use of avidin-biotin-peroxidase complex $(\mathrm{ABC})$ in immunoperoxidase techniques. A comparison between $\mathrm{ABC}$ and unlabeled antibody (PAP) procedures. J. Histochem. Cytochem., 29, 577-588.

2) Muto, T. \& Adachi, S. (1988) Development of colonic carcinoma: Clinical and histopathological observations. Pathol. Clin. Med., 6, 1025-1034. (Japanese) 